\title{
Creating the Illusion of Sportiness: Evaluating Modified Throttle Mapping and Artificial Engine Sound for Electric Vehicles
}

\author{
Timo Melman $\mathbb{D}^{1,2,3}$ Peter Visser, ${ }^{1,2}$ Xavier Mouton, ${ }^{2}$ and Joost de Winter $\mathbb{D}^{1}$ \\ ${ }^{1}$ Department of Cognitive Robotics, Faculty of Mechanical, Maritime and Materials Engineering, Delft University of Technology, \\ Mekelweg 2, 2628 CD Delft, Netherlands \\ ${ }^{2}$ Group Renault, Chassis Systems Department, 1 Avenue du Golf, 78280 Guyancourt, France \\ ${ }^{3}$ Department of Computer and System Engineering, ENSTA ParisTech, 828 Boulevard des Maréchaux, \\ 91762 Palaiseau Cedex, France
}

Correspondence should be addressed to Joost de Winter; j.c.f.dewinter@tudelft.nl

Received 25 May 2021; Revised 28 August 2021; Accepted 11 September 2021; Published 15 October 2021

Academic Editor: Stefano de Luca

Copyright (C) 2021 Timo Melman et al. This is an open access article distributed under the Creative Commons Attribution License, which permits unrestricted use, distribution, and reproduction in any medium, provided the original work is properly cited.

\begin{abstract}
Modern computerized vehicles offer the possibility of changing vehicle parameters with the aim of creating a novel driving experience, such as an increased feeling of sportiness. For example, electric vehicles can be designed to provide an artificial sound, and the throttle mapping can be adjusted to give drivers the illusion that they are driving a sports vehicle (i.e., without altering the vehicle's performance envelope). However, a fundamental safety-related question is how drivers perceive and respond to vehicle parameter adjustments. As of today, human-subject research on throttle mapping is unavailable, whereas research on sound enhancement is mostly conducted in listening rooms, which provides no insight into how drivers respond to the auditory cues. This study investigated how perceived sportiness and driving behavior are affected by adjustments in vehicle sound and throttle mapping. Through a within-subject simulator-based experiment, we investigated (1) Modified Throttle Mapping (MTM), (2) Artificial Engine Sound (AES) via a virtually elevated rpm, and (3) MTM and AES combined, relative to (4) a Baseline condition and (5) a Sports car that offered increased engine power. Results showed that, compared to Baseline, AES and MTM-AES increased perceived sportiness and yielded a lower speed variability in curves. Furthermore, MTM and MTM-AES caused higher vehicle acceleration than Baseline during the first second of driving away from a standstill. Mean speed and comfort ratings were unaffected by MTM and AES. The highest sportiness ratings and fastest driving speeds were obtained for the Sports car. In conclusion, the sound enhancement not only increased the perception of sportiness but also improved drivers' speed control performance, suggesting that sound is used by drivers as functional feedback. The fact that MTM did not affect the mean driving speed indicates that drivers adapted their "gain" to the new throttle mapping and were not susceptible to risk compensation.
\end{abstract}

\section{Introduction}

Drivers use their vehicles as more than just a means to arrive at their destinations. As explained by Rothengatter [1]; road user behavior is to an extent governed by the "pleasure of driving fast" (p. 605). Indeed, a portion of road users appears to be attracted to sporty driving, as evidenced by the sales of sports cars or vehicle models that offer high engine power and agile handling characteristics [2]. As an alternative, several manufacturers produce vehicles that can provide a sporty driving experience via a sport mode the driver can select. The sport mode has gained a substantial presence on the car market today [3-8].
According to manufacturers, the sport mode "permits an increased responsiveness from the engine and the gearbox" [7] and offers a "sporty driving style" [5]. The sport mode may encompass technology that increases the throttle sensitivity, road holding, and agility of the vehicle [9-11]. This includes the active drivetrain, for example, changes in engine mapping and gear shifting $[12,13]$, active suspension, and four-wheel steering $[14,15]$. Additionally, sport modes can be accompanied by mechanical sound enhancement, which concerns the adjustment of physical elements of the drivetrain and the active control of valves that redirect the engine airflow and influence the exhaust sound [16, 17]. 
In recent decades, several techniques have been developed to increase perceived sportiness without altering the vehicle dynamics and without requiring costly components or mechanical adjustments to the vehicle. Two of such techniques are Artificial Engine Sound and Modified Throttle Mapping.

1.1. Artificial Engine Sound (AES). Artificial Engine Sound (AES) refers to a system that produces synthetic sounds through the cabin speakers. AES has been proposed for electric vehicles (e.g., [18-22]). However, current research on sounds for electric vehicles mostly focuses on pedestrian safety (e.g., $[23,24])$. Considerably less research is available that focuses on the experience of drivers inside the electric vehicle.

Psychoacoustics research has shown that perceived sportiness can be increased by adjusting characteristics of the sounds, such as loudness, roughness, sharpness, and tonality $[25,26]$. However, a limitation of psychoacoustics studies is that they are typically conducted in listening rooms. As Jennings et al. ([27], (p. 1263)) argued, "perception of the sounds of on-road cars is affected by stimuli for other senses (e.g., visual and vibrational), and the fact that an assessor is also concentrating on driving." To illustrate, research in a listening room by Park et al. [28] found that loudness was predictive of perceived sportiness $(r=0.84)$ but negatively predictive of perceived comfort $(r=-0.83)$, consistent with the generally accepted "trade-off hypothesis of pleasantness and power" ([29] p. 1203). A driving simulator study by Hellier et al. [30], however, found that drivers regarded no engine noise at all as uncomfortable. Hence, it appears that sound perception may be different in listening rooms as compared to active driving.

Very little research on perceived sportiness in real vehicles is available. An exception is Zeitler and Zeller [31], who let acoustical experts rate the interior sounds of different vehicles on a test track. Their results showed that perceived sportiness was strongly correlated with the sound volume increase during engine load (i.e., while accelerating). However, engine performance (e.g., actual sportiness) and acoustic feedback were confounded; that is, the vehicles that delivered more power were also those that produced a sporty sound. In a follow-up experiment, they tried to disentangle these two effects using AES and found that vehicle sounds and engine torque independently contributed to perceived sportiness.

Apart from investigating the effects of AES on perceived sportiness, it is essential to examine the extent to which AES influences driving behavior. Previous research suggests that the presence and volume of vehicle sound affect driving speeds. More specifically, it has been found that a reduction in engine volume or the lack of engine sound causes drivers to drive faster [30, 32], underestimate their speed [32-34], and show poorer speed control [35-37]. These findings are consistent with the notion that engine sound acts as an information source that facilitates perception and control, or as argued by Hellier et al. ([30] p. 598), "engine noise can be characterised as "feedback" rather than "noise."”
In summary, although the above-mentioned studies indicate that the presence and volume of sound affect driving behavior, there appears to be a lack of research about how drivers perceive and respond to sound enhancement techniques that could be applied in electric vehicles, such as AES. Furthermore, research on vehicle sound has to date been predominantly conducted in listening rooms, a setting that cannot provide information about drivers' speed adaptation.

1.2. Modified Throttle Mapping (MTM). A second approach that may increase perceived sportiness without requiring mechanical components is Modified Throttle Mapping (MTM). MTM is defined as the software-based adjustment of the relationship between the driver's throttle input and the engine throttle input. Through MTM, for a given driver throttle input, the engine produces more torque while the maximum torque (i.e., the torque for $100 \%$ driver throttle input) remains the same. Note that MTM is not the same as modified "engine mapping," that is, the adjustment of engine characteristics through changes in fuel injection, air charge, ignition timing, and valve timing and other factors that influence engine performance $[38,39]$.

Research describes different ways of changing the throttle mapping and the corresponding effect on vehicle performance (e.g., $[10,40,41])$, but only a few studies have investigated the effects of MTM on driving behavior. The few studies that did investigate human-in-the-loop effects of MTM used intelligent controllers, such as a throttle pedal for regulating the desired engine torque and desired wheel torque [42] or a throttle pedal that caused the vehicle to decelerate more strongly upon releasing the pedal in critical car-following situations [43].

1.3. Aim and Hypotheses. Little is known about how drivers perceive and respond to vehicle parameter adjustments that intend to provide a sporty driving experience for electric vehicles, such as MTM and AES technology. It is important to investigate this topic with a view to road safety. If such systems reduce vehicle controllability and increase driving speed, this could be seen as undesirable.

The current study aimed to investigate how drivers perceive and respond to AES and MTM, two systems that intend to provide a sporty driving experience for electric vehicles and do not change the vehicle's performance envelope in any way. The individual and combined contributions of MTM and AES were compared to a Baseline condition and a vehicle that offered increased engine power ("Sports car"). The Sports car was included to investigate how the results for AES and MTM compare to a car that offers actually increased sportiness. The combined condition (AES-MTM) was included to examine whether or not the effects of MTM and AES are additive.

The expected effects of MTM and AES can be explained using theory from the field of manual control (e.g., [44]). Figure 1 shows a model of human driving behavior in a speed control task, based on Weir and Chao [45] and McRuer et al. [46]. Here, the human outputs a foot movement ("throttle driver"), which via the throttle 


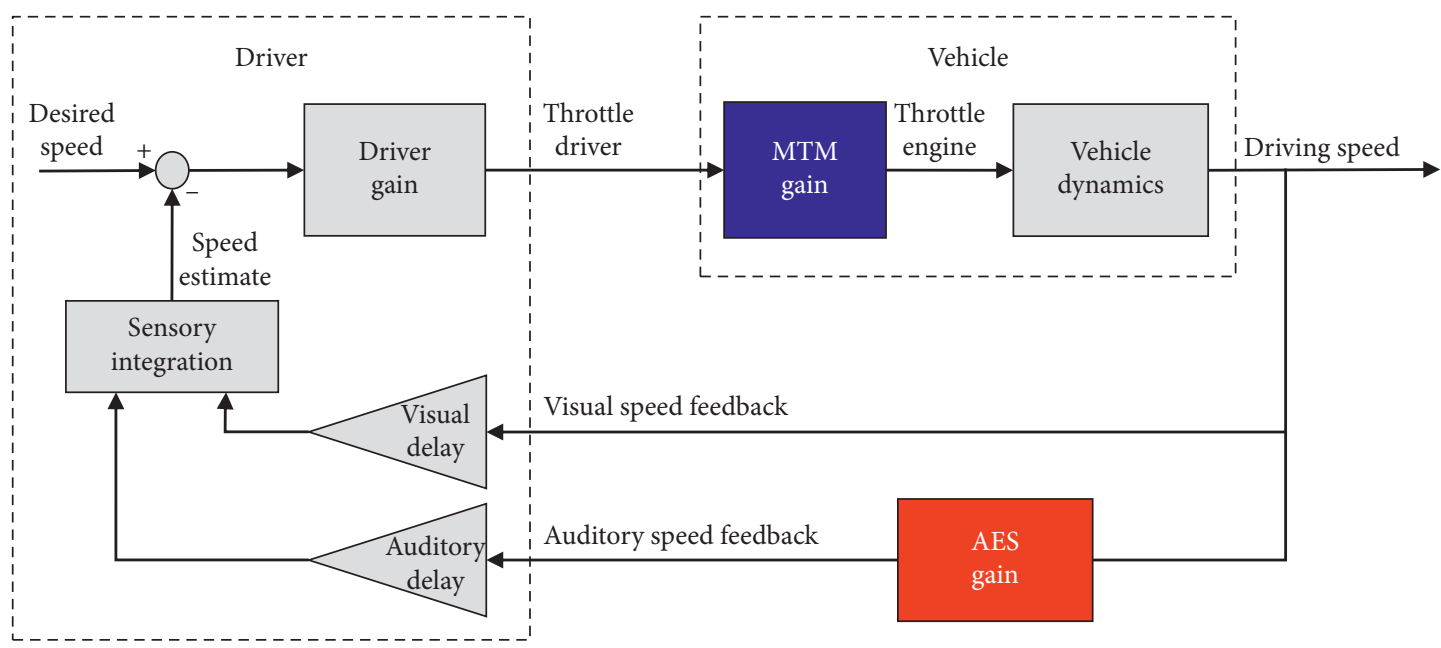

FIgURE 1: Schematic representation of a driver driving with Modified Throttle Modification (MTM) and Artificial Engine Sound (AES). This figure assumes a fixed-base driving simulator, which means that the driver is not provided with physical motion feedback.

mapping (a variable gain, i.e., a multiplication factor) results in an input to the vehicle model ("throttle engine," describing how much torque is requested from the car). The car model outputs the current driving speed, which is fed back to the driver via visual and auditory pathways. The driver perceives these two feedback sources with a time delay. Additionally, the driver is represented by a gain, which describes how strongly the driver responds to the difference between the perceived speed and the desired speed. The desired speed represents the speed at which the driver wishes to drive at a particular moment; it is dependent on many factors, including the environment (road curvature; road width), the driver's personality, and the driver's risk assessment based on the visual and auditory information received.

If drivers apply a particular "throttle driver," this will result in a higher "throttle engine" when driving with MTM (i.e., high MTM gain) compared to without (i.e., low MTM gain). Accordingly, MTM was hypothesized to increase perceived sportiness and vehicle acceleration compared to without. However, it can be expected that the effect of MTM on driving speed is only short-lasting, as visual feedback is dominant in driving (e.g., [47]). This hypothesis can be further motivated from the viewpoint of open-loop versus closed-loop control [48]. That is, MTM is expected to increase vehicle acceleration (and thus speed) for short-term "open-loop" leg movements involved in pressing the pedal but will not affect "closed-loop" driving speed. Note that Figure 1 depicts a closed-loop situation, where the driver uses auditory and visual feedback to control the car's speed. In the context of Figure 1, an increase of MTM gain is expected to result in a reciprocal decrease in the driver gain, so that the driving speed is unaffected.

In the current study, AES was implemented through an increase of the "virtual rpm," where we assumed an electric vehicle that generated the sound of a combustion engine. When driving with AES enabled (i.e., high AES gain), fluctuations in driving speed result in larger fluctuations in engine sound pitch as compared to Baseline (i.e., a condition with a low AES gain). In other words, the enabling of AES can be expected to strengthen the auditory feedback loop, making drivers better aware of speed fluctuations and thus contributing to a more accurate estimate of driving speed relative to the desired driving speed (see [30] for a similar argumentation regarding vehicle sound in general). It is noted that visual feedback is processed relatively slowly compared to the other sensory modalities [49]. This may be especially true when it comes to the perception of longitudinal ego-speed, which requires the driver to extract (changes in) optical flow and edge rate information [50] (but note that the speedometer provides a more direct indication of speed). Hence, auditory feedback may have an important role in speed control.

In addition to the mechanisms depicted in Figure 1, AES may influence the desired driving speed itself. As pointed out above, increasing the engine sound volume leads to a lower driving speed. In the same vein, the increased virtual rpm can be expected to result in lower driving speeds. This hypothesis is consistent with the phenomenon of risk compensation, which predicts that drivers increase their speed when provided with protective or assistive technology or conversely reduce their speed when provided with technology that reduces protection or increases the perceived risk [51-53].

The hypothesized effects for MTM and AES are summarized in Table 1.

\section{Method}

2.1. Participants. Thirty-two participants (6 females) between 19 and 35 years old $(M=23.4, S D=3.1)$ with normal or corrected-to-normal vision volunteered for the driving simulator experiment. Regarding the question "On average, how often did you drive a vehicle in the last 12 months," 3 participants reported every day, 5 reported 4 to 6 days a week, 12 reported 1-3 days a week, 11 reported once a month to once a week, and 1 reported never. Regarding mileage in the past 12 months, the most frequently selected 
TABLE 1: Hypothesized effects of the two systems on perceived sportiness and driving behavior relative to a Baseline condition.

\begin{tabular}{lcc}
\hline & Perceived sportiness & Driving speed \\
\hline Artificial Engine Sound (AES) & Higher & Lower mean and lower variability \\
Modified Throttle Mapping (MTM) & Higher & Higher (short term) \\
\hline
\end{tabular}

response category was $1001-5000 \mathrm{~km}$ (14 respondents), followed by $5001-10000 \mathrm{~km}$ (7 respondents), $10001-15000 \mathrm{~km}$ (4 respondents), and $15001-20000 \mathrm{~km}(4$ respondents). The research was approved by the Human Research Ethics Committee (HREC) of the Delft University of Technology, and all participants provided written informed consent.

2.2. Apparatus. The experiment was conducted in a fixedbase simulator (see Figure 2). The steering wheel and pedals used in the simulation were from a Sensodrive SENSOWheel running at $1 \mathrm{kHz}$. The simulation was developed using JOAN [54], an open-source software framework developed at the TU Delft that builds on the CARLA opensource simulator (Version 0.9.8 [55]). The vehicle dynamics were simulated by the Unreal Engine using PhysX [56]. The scenery was shown via a 65 -inch $4 \mathrm{k}$ TV with a $60 \mathrm{~Hz}$ refresh rate. The virtual camera settings provided a 90-degree horizontal field of view. The simulation and data logging were updated at $100 \mathrm{~Hz}$. The sound of the vehicle's engine was presented via Beyerdynamic DT-770 Pro 32 Ohm headphones. The car's interior and bonnet were included in the visualization to enhance the perception of road position and vehicle speed and were the same for all conditions (Figure 2). The vehicle speed was shown digitally in light blue font above the bonnet.

2.3. Independent Variables and Design. Participants drove five trials, with one of the following five conditions per trial:

(1) Baseline

(2) Artificial Engine Sound (AES)

(3) Modified Throttle Mapping (MTM)

(4) Modified Throttle Mapping and Artificial Engine Sound Combined (MTM-AES)

(5) Sports car

The five conditions were presented according to a balanced within-subject design (Williams design).

For the Baseline, AES, MTM, and MTM-AES conditions, the simulated vehicle was a $2 \mathrm{~m}$ wide sedan of $2316 \mathrm{~kg}$. It had a maximum engine torque of $350 \mathrm{Nm}$, a maximum speed of $149 \mathrm{~km} / \mathrm{h}$, and a drag coefficient of 0.24 . The vehicle was modeled after an electric vehicle, with a single-gear gearbox and a nearly constant engine torque for different speeds. The Sports car offered increased engine power. It was modeled after the same heavy sedan and visually identical to the other four conditions, but with an increased maximum torque of $550 \mathrm{Nm}$ and a maximum speed of $203 \mathrm{~km} / \mathrm{h}$.

For the Baseline, AES, MTM, and MTM-AES conditions, the same interior sound of a vehicle driving at constant

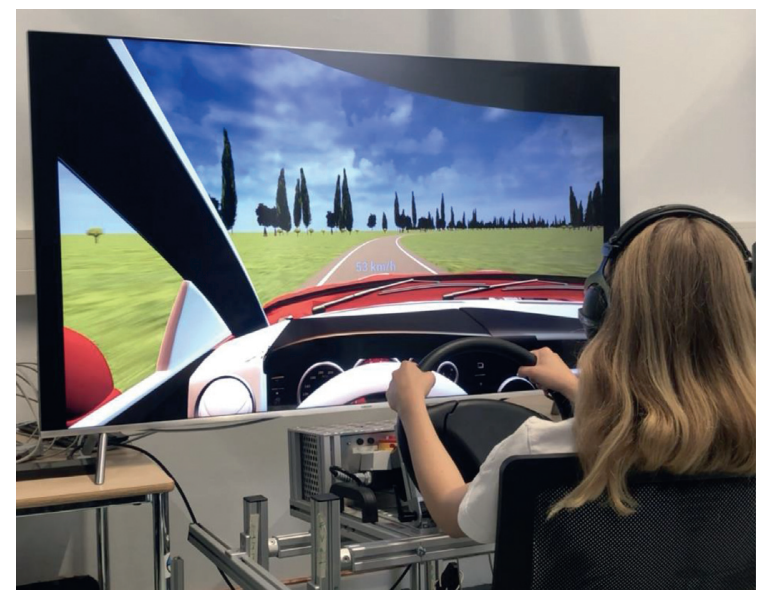

FIgURE 2: The experimental environment with a participant driving in the fixed-based simulator. The digital speedometer can be seen in light blue font, just above the bonnet.

speed was used (Volkswagen Golf V 1.6 FSI running at $2140 \mathrm{rpm}$ [57]). This sound was looped, and the playback speed of the interior sound was slowed down or sped up depending on the vehicle speed and momentary engine torque ("throttle engine"), hence providing a virtual rpm. In this way, the vehicle sound was informative about vehicle speed and engine torque. The Sports car had a higher rpm and was based on another sound sample (Audi A4 B8 20TDI running at $3050 \mathrm{rpm}$ [58]), which produced a more racy sound at high virtual rpm. Figure 3 shows how the virtual $\mathrm{rpm}$ depended on vehicle speed and engine torque, for Baseline, AES, and the Sports car. The mapping shown in Figure 3 was based on extensive pilot testing, where it was made sure that the differences between conditions were noticeable and within a realistic rpm range.

The MTM condition changed the relationship between the driver's throttle depression ("throttle driver") and the normalized requested engine torque ("throttle engine"), without affecting the maximum engine power (Figure 4). For a "throttle driver" value of $27 \%$, the difference in "throttle engine" between Baseline (15\%) and MTM (49\%) was maximal (34\%). The throttle mappings were based on pilot tests, where it was made sure that the difference between Baseline and MTM was noticeable while retaining controllability. For the Sports car, a linear throttle mapping was used, where $0 \%$ "throttle driver" corresponded to $0 \%$ "throttle engine" and 100\% "throttle driver" corresponded to $100 \%$ "throttle engine."

2.4. Road Environment. The participants drove five trials on the same single-lane road ( $3.6 \mathrm{~m}$ wide and $8.1 \mathrm{~km}$ long). Trees, buildings, landscapes, and guardrails were placed next 


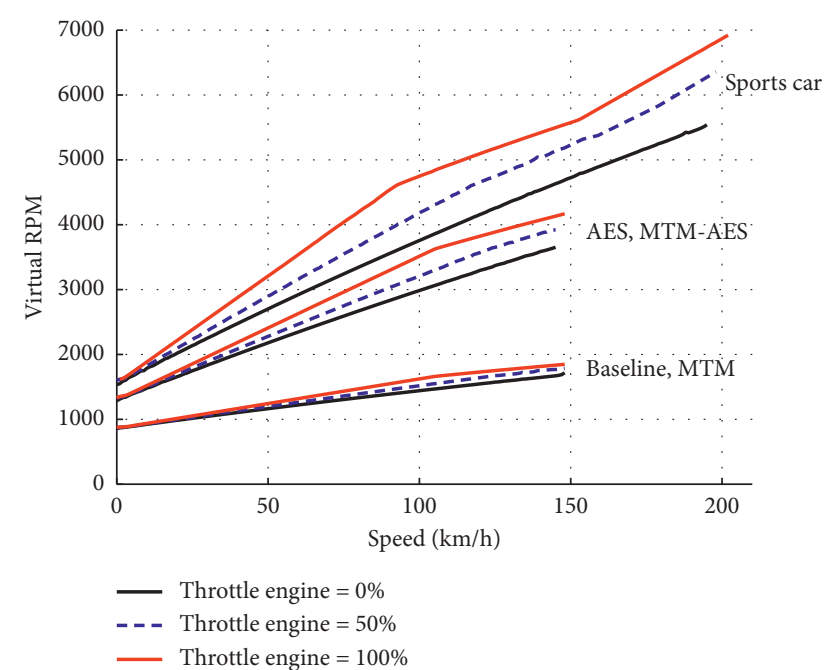

FIGURE 3: Virtual engine speed (rpm) as a function of vehicle speed and throttle engine. The data used to create this graph were obtained from the data recordings of the experiment.

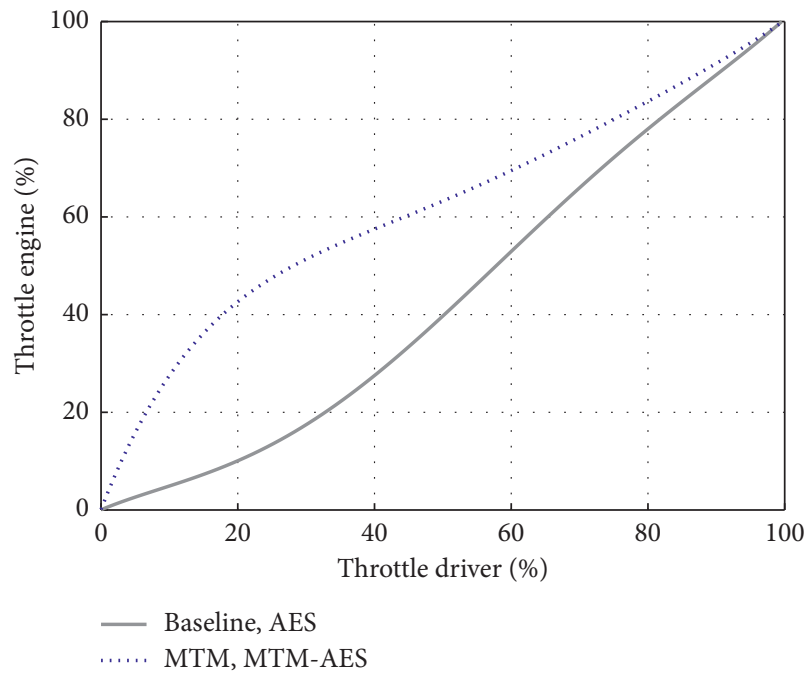

Figure 4: The Modified Throttle Mapping. "Throttle driver" was measured by a displacement sensor attached to the throttle pedal, whereas "throttle engine" was determined by software. Note that "throttle driver" could become slightly smaller than 0 when releasing the pedal or exceed $100 \%$ when fully depressing the pedal. In the analysis, all values above 100 and below 0 were rounded to 100 and 0 , respectively. The "throttle driver" range from 0 to $100 \%$ corresponded to a physical pedal depression of about $75 \mathrm{~mm}$ at an exerted force of about $35 \mathrm{~N}$.

to the road. The route was divided into an acceleration section, a straight section, and a curvy section (Figure 5).

The first $2.6 \mathrm{~km}$ consisted of an acceleration task where drivers were requested to accelerate four times to $60 \mathrm{~km} / \mathrm{h}$. The locations of the acceleration were indicated via stop signs and speed limit signs next to the road. The acceleration section was implemented to ensure that all drivers strongly accelerated at least four times per condition. The middle $2 \mathrm{~km}$ was a straight section where participants could choose

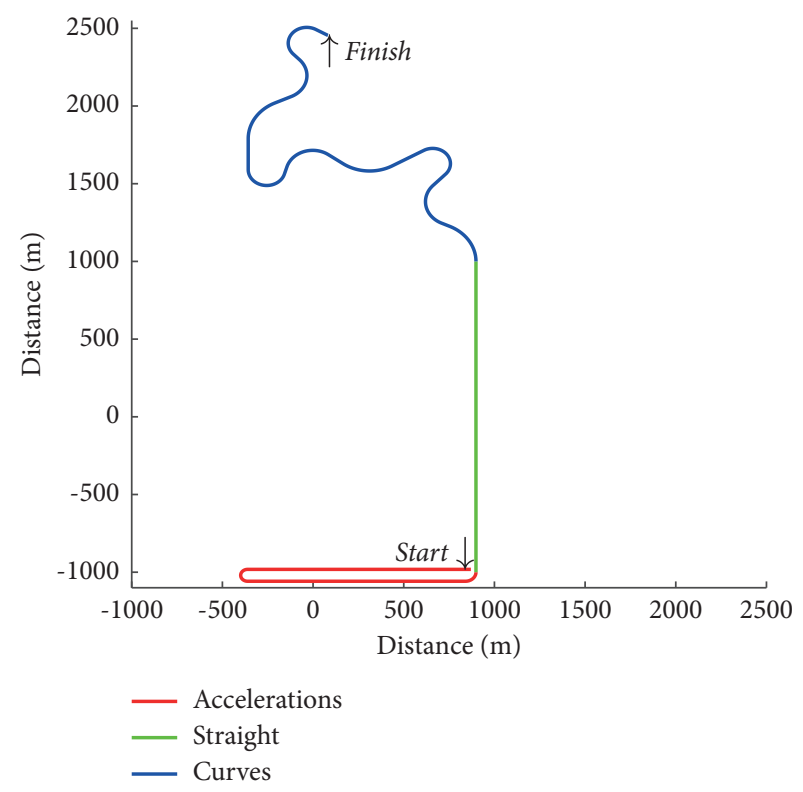

FIgURE 5: Top view of the $8.1 \mathrm{~km}$ driven route consisting of three sections: an acceleration section, a straight section, and a curvy section.

a speed at which they felt comfortable. The straight allowed an investigation of the participants' speed choice.

The last $3.5 \mathrm{~km}$ consisted of a curvy section that contained curves with an inner radius of 100, 150, and $250 \mathrm{~m}$. Each curve type appeared three times, and the curves were connected by straight sections with a length of 50 or $100 \mathrm{~m}$. No traffic and no on-road obstacles were simulated. The curvy section allowed investigating naturalistic deceleration and acceleration for curves.

2.5. Procedure. Participants first read and signed a consent form and completed a questionnaire regarding their demographics and driving experience. Participants were asked to drive as they usually would and adhere to the traffic rules identified by road signs next to the road. Next, the participants were requested to sit in the simulator.

Before each condition, a three-minute training run was performed on a road consisting of straights and curves. During each training trial, the participants were asked to familiarize themselves with the upcoming condition by accelerating, decelerating, and curve driving.

In each experimental trial, participants drove in one of the five conditions (Baseline, MTM, AES, MTM-AES, or Sports car). After each trial, participants stepped out of the simulator and completed a questionnaire about their driving experience. The experiment took approximately 75 minutes per participant.

2.6. Dependent Measures. A distinction is made between self-reported experience and driving behavior.

Self-reported experience: after each trial, participants completed a questionnaire containing 14 questions on a fivepoint scale. The first four questions investigated drivers' 
perceived effort for Q1 braking, Q2 steering, Q3 accelerating, and Q4 maintaining speed, on a scale of low to high. This was followed by three questions regarding the perception of the vehicle: Q5 engine responsiveness (low to high), Q6 brake responsiveness (high to low), and Q7 engine sound of the vehicle (high to low).

Finally, seven questions were asked in which participants had to answer whether they had experienced the vehicle as Q8 sporty/not sporty, Q9 dangerous/safe, Q10 comfortable/not comfortable, Q11 undesirable/desirable, Q12 raising alertness/sleep-inducing, Q13 irritating/likable, and Q14 sluggish/ quick. In the analysis, answers to Q6, Q7, Q8, and Q10 were mirrored such that the results were expressed on a scale from low to high.

Of note, Q8 was the main question of interest in this research (i.e., perceived sportiness), whereas Q10, Q11, and Q13 assessed acceptance (Q11 and Q13 were from the satisfaction dimension of an acceptance survey of [59]). Q1, Q2, and Q6 were negative control questions that were expected not to be affected by any of the experimental conditions, whereas Q3, Q5, Q7, and Q14 were positive control questions, expected to be affected by at least one of the experimental conditions. Finally, Q4, Q9, and Q12 assessed additional experiences of interest, namely, whether participants found it difficult to control the speed of the vehicle (Q4), whether they found the vehicle safe (Q9), and how the experimental conditions affected their overall arousal level (Q12).

2.7. Principal Component Analysis on the Questionnaire Results. To identify underlying dependencies and to increase the interpretability of the 14 questionnaire items, a principal component analysis (PCA) was performed. A matrix with responses for 160 trials (32 participants $\mathrm{x} 5$ trials per participant) and 14 items was normalized to a mean of 0 and a standard deviation of 1 and submitted to PCA. The first two components were retained, and component scores per participant and experimental condition were computed after oblique rotation (Promax):

2.7.1. Driving Behavior. The driving behavior measures were calculated separately for the straight and curvy sections (Figure 5). For the straight section, the first $800 \mathrm{~m}$ and last $400 \mathrm{~m}$ were discarded to investigate steady-state driving. For the curvy section, the entire $3.5 \mathrm{~km}$ was used. The following measures were calculated:

(i) Mean speed $(\mathrm{km} / \mathrm{h})$ : a measure for road safety. An increase in speed reduces a driver's time to respond in an emergency scenario and increases the probability of being involved in a crash $[60,61]$.

(ii) Standard deviation (SD) of the speed $(\mathrm{km} / \mathrm{h})$ : a higher $S D$ speed indicates that drivers were not able or willing to maintain a constant speed.

(iii) Mean absolute longitudinal acceleration $\left(\mathrm{m} / \mathrm{s}^{2}\right)$ : this is a measure indicative of the "fluency" and sportiness of driving. A high mean absolute longitudinal acceleration can be seen as sporty driving behavior $[62,63]$.

(iv) Mean throttle driver (\%): a measure of how deeply drivers pressed the accelerator on average.

(v) Mean throttle engine (\%): this measure indicates how much engine torque was delivered on average. Together, "throttle driver" and "throttle engine" allowed examining how drivers adapted to the MTM condition.

(vi) Throttle driver released time (\%): in the literature, this measure is also referred to as coasting. Coasting has been interpreted as indicative of uncertainty or a delay in decision-making $[64,65]$. It can also be seen as a consequence of having accelerated too much, resulting in an overshoot of speed and a subsequent throttle release.

Additionally, an analysis was performed for the acceleration section, where the first second of the four acceleration phases (i.e., accelerating from standstill) was averaged per experimental condition. The start of each trial was determined based on the moment the throttle position exceeded $0 \%$.

2.8. Statistical Analyses. For each measure, a matrix of $32 \times 5$ numbers was obtained (32 participants and 5 conditions). For each condition in this matrix, the mean, standard deviation $(S D)$, and $95 \%$ within-subject confidence interval (CI) were computed. The $95 \%$ within-subject confidence intervals were calculated according to Morey (2008), where the matrix was normalized by subtracting for each participant the mean of all conditions from the five condition observations before using the standard method for determining the $95 \% \mathrm{CI}$. The CI was adjusted with a correction factor based on the number of experimental conditions [66]. According to Cumming and Finch [67], nonoverlapping CIs correspond to a $p$-value smaller than 0.006 .

\section{Results}

3.1. Self-Reported Experience. Figure 6 shows the means and within-subject $95 \%$ confidence intervals for the 14 questionnaire items. Compared to Baseline, AES and MTM-AES resulted in increased perceived sportiness (Q8), perceived engine responsiveness (Q5), and perceived quickness (Q14). Furthermore, AES and MTM-AES were clearly perceived overall (Q7), whereas desirability (Q11) and likability (Q13) did not show significant differences from Baseline.

MTM-AES resulted in an increased self-reported effort to maintain speed compared to AES, and a similar trend was evident for MTM versus Baseline (Q4). The effects of MTM on the other questionnaire items, including sportiness (Q8), were not significant. For the three negative control questions (brake effort (Q1), steering effort $(\mathrm{Q} 2)$, and brake response (Q6)), no significant differences were observed between the five conditions.

The Sports car yielded lower acceleration effort (Q3), higher perceived engine responsiveness (Q5), and higher perceived sportiness $(\mathrm{Q} 8)$ than the other four conditions. Additionally, the Sports car was regarded as less sleep- 

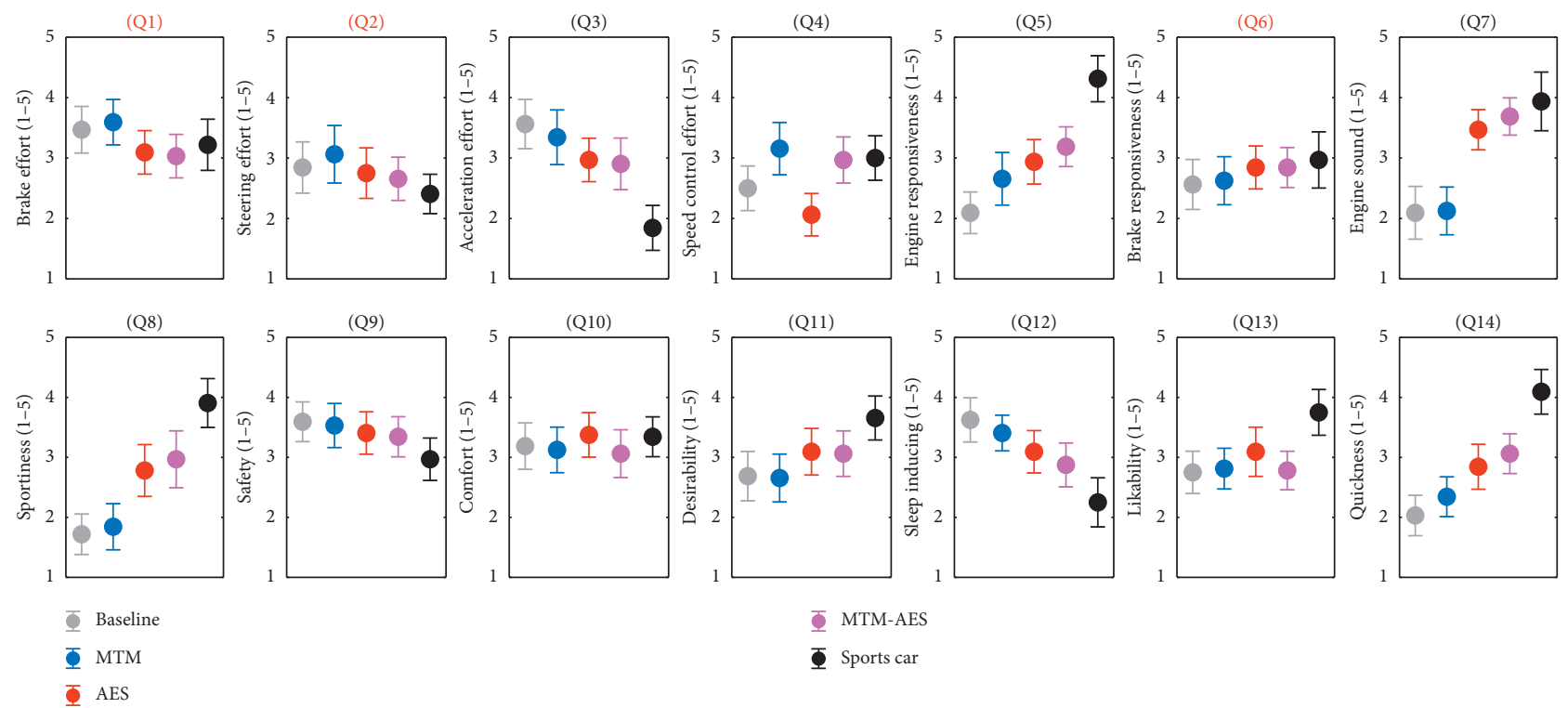

FIGURE 6: The questionnaire results for the 14 questions for the five conditions. The figure depicts the means (circles) and within-subject 95\% confidence intervals (whiskers). The questionnaire numbers for the negative control questions (Q1, Q2, and Q6) are indicated in red.

inducing (Q12) and quicker (Q14). Finally, the Sports car was liked (Q13) and regarded as desirable (Q11).

The principal component analysis loadings are shown in Table 2. The first principal component is primarily composed of questions related to sportiness and acceleration of the vehicle. The second principal component is mainly composed of questions that correspond to comfort, safety, likability, and desirability. In total, the first two principal components captured $53.2 \%$ of the variance (PC1-Sportiness: 36.8\%; PC2-Positive Affect: 16.4\%).

The mean PC1-Sportiness scores (95\% CI) for Baseline, MTM, AES, MTM-AES, and Sports car were $-0.813,[-1.083$, $-0.543],-0.542[-0.824,-0.261], 0.000[-0.256,0.256], 0.201$ $[-0.078,0.480]$, and $1.153[0.874,1.432]$. The mean PC2Positive Affect scores (95\% CI) for Baseline, MTM, AES, MTM-AES, and Sports car were 0.078 [-0.291, 0.447], -0.156 $[-0.542,0.230], 0.196[-0.167,0.559],-0.187[-0.513,0.139]$, and $0.069[-0.198,0.336]$. Thus, the PC1-Sportiness scores were substantially elevated for the Sports car, as well as the AES and MTM-AES conditions, whereas no clear differences between the five conditions were found in PC2-Positive Affect.

3.2. Driving Behavior. Figure 7 shows the road curvature, driving speed, throttle driver, and throttle engine averaged over all participants as a function of traveled distance. It can be seen that participants adhered to the traffic signs and accelerated four times to $60 \mathrm{~km} / \mathrm{h}$ (in accordance with the instructions) and adopted an average speed during the straight section of $115-120 \mathrm{~km} / \mathrm{h}$. In the subsequent curvy section, drivers can be seen to slow down more for sharper curves. Over the entire track, drivers adopted lower throttle inputs when driving with MTM and MTM-AES compared to Baseline and AES.
TABle 2: Promax-rotated PCA loadings for the first two principal components (PC).

\begin{tabular}{lcc}
\hline & $\begin{array}{c}\text { PC1- } \\
\text { Sportiness }\end{array}$ & PC2-Positive Affect \\
\hline Q1. Brake effort & 0.00 & -0.26 \\
Q2. Steering effort & -0.16 & -0.52 \\
Q3. Acceleration effort & -0.78 & -0.15 \\
Q4. Speed control effort & 0.09 & -0.62 \\
Q5. Engine responsiveness & 0.83 & 0.02 \\
Q6. Brake responsiveness & -0.08 & 0.01 \\
Q7. Engine sound & 0.50 & -0.24 \\
Q8. Sportiness & 0.83 & 0.08 \\
Q9. Safety & -0.35 & 0.72 \\
Q10. Comfort & 0.02 & 0.77 \\
Q11. Desirability & 0.41 & 0.64 \\
Q12. Sleep-inducing & -0.78 & 0.19 \\
Q13. Likability & 0.44 & 0.64 \\
Q14. Quickness & 0.87 & 0.13 \\
\hline
\end{tabular}

3.3. Effects of MTM (MTM versus Baseline and MTM-AES versus AES). Table 3 shows the means, standard deviations, and nonoverlapping confidence intervals for the driving behavior measures. Figure 7 and Table 3 show that, for both sections, participants applied a significantly lower "throttle driver" for MTM compared to Baseline and AES, whereas no significant differences were found for "throttle engine." In other words, participants adapted to the MTM condition by pressing the throttle less deeply. Furthermore, for MTM and MTM-AES, the throttle-released time was about twice as high as Baseline and AES. The results for the first second of the acceleration phase (i.e., accelerating from standstill) showed a higher mean acceleration for MTM and MTM-AES compared to Baseline and AES. 


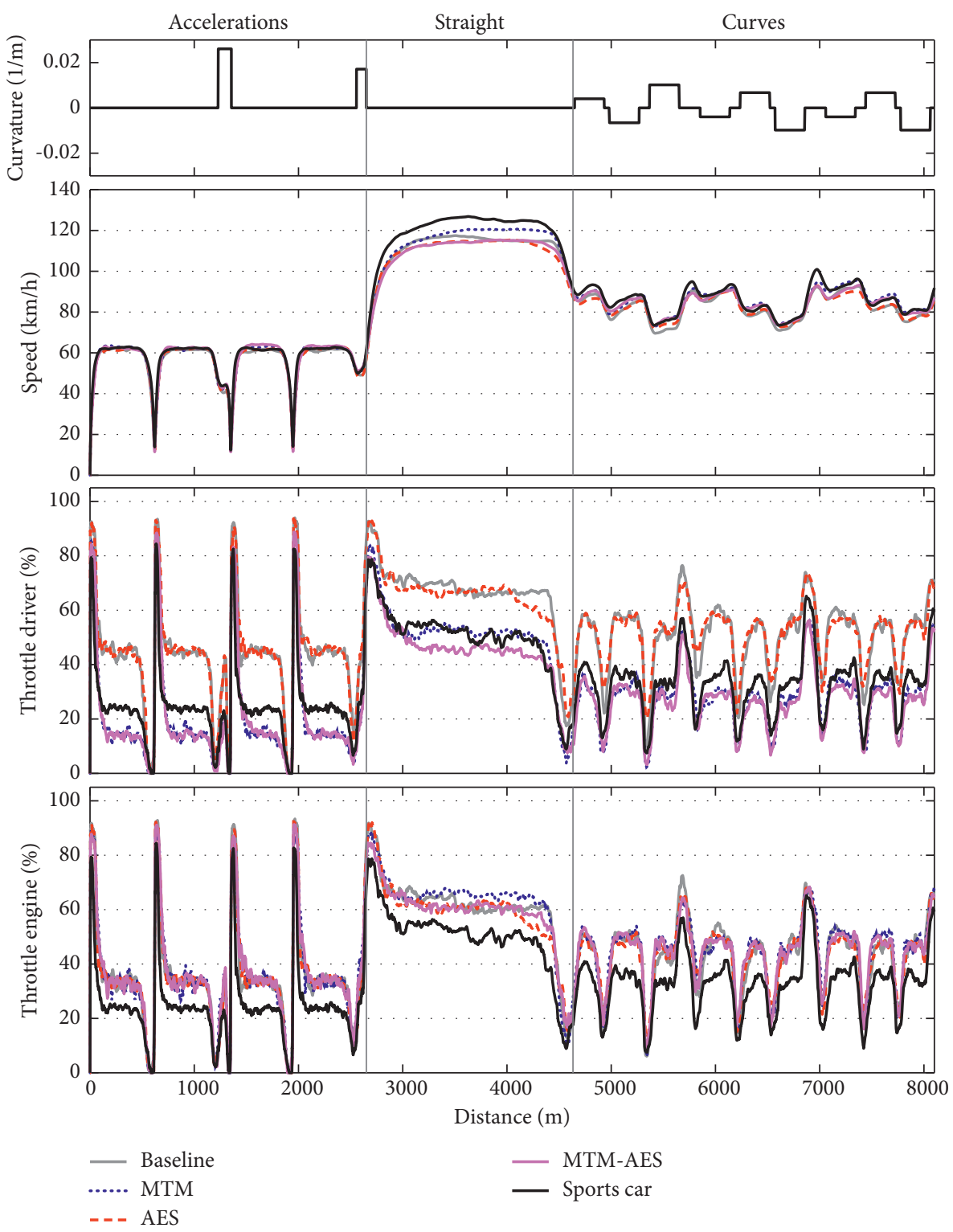

FIGURE 7: The mean driving behavior per condition averaged over all 32 participants, from top to bottom: road curvature, speed, acceleration, throttle driver, and throttle engine. The vertical lines demarcate the acceleration, straight, and curvy sections. Note that the vehicle speed does not drop to exactly $0 \mathrm{~km} / \mathrm{h}$ during the acceleration section; this is because participants stopped their vehicle at slightly different distances on the road.

Figure 8 depicts the distribution for "throttle driver" and "throttle engine" for all participants combined for the curvy section. It can again be seen that, compared to Baseline and AES, MTM and MTM-AES resulted in substantially lower "throttle driver" values (Figure 8, top). For "throttle engine," the mean was equivalent between conditions (see Table 3), but the distribution of "throttle engine" (Figure 8, bottom) showed clear differences between conditions. More specifically, for MTM and MTM-AES, low "throttle engine" levels (5-40\%) were underrepresented, and high "throttle engine" levels (40-75\%) were overrepresented compared to Baseline and AES. For the Sports car, a lower "throttle engine" was found compared to the other conditions. This can be explained by the fact that the Sports car had more engine power, and thus, a lower "throttle engine" was needed to drive at a particular speed (see also Table 3).

Figure 9 shows the participants' mean acceleration during the first $3 \mathrm{~s}$ after driving away. It shows that drivers adopted significantly higher acceleration when driving with MTM and MTM-AES than Baseline and AES. The increased acceleration for MTM compared to Baseline, which was described above, is clearly visible. After about $1.5 \mathrm{~s}$, the acceleration was equivalent for Baseline, MTM, AES, and MTM-AES conditions.

3.4. Effects of AES (AES versus Baseline and AES versus MTM). Table 3 shows that no significant effects of AES on mean driving speed were found. However, AES resulted in a 


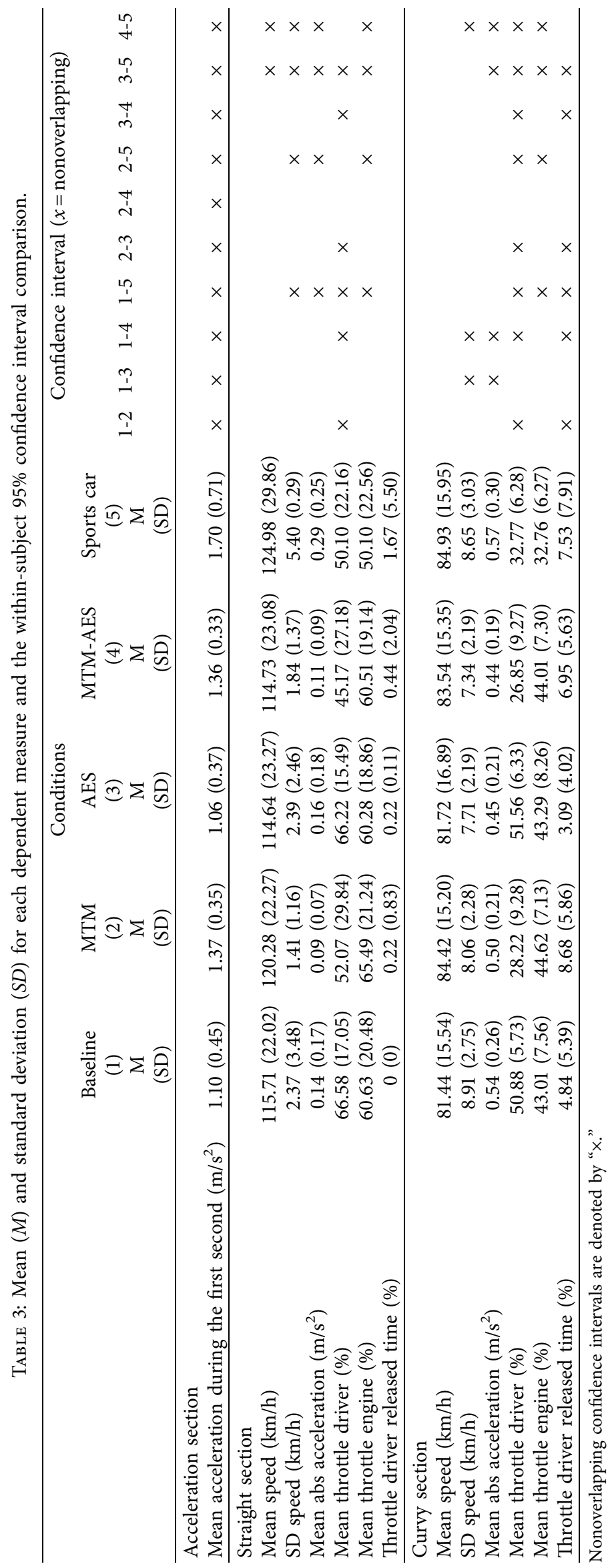



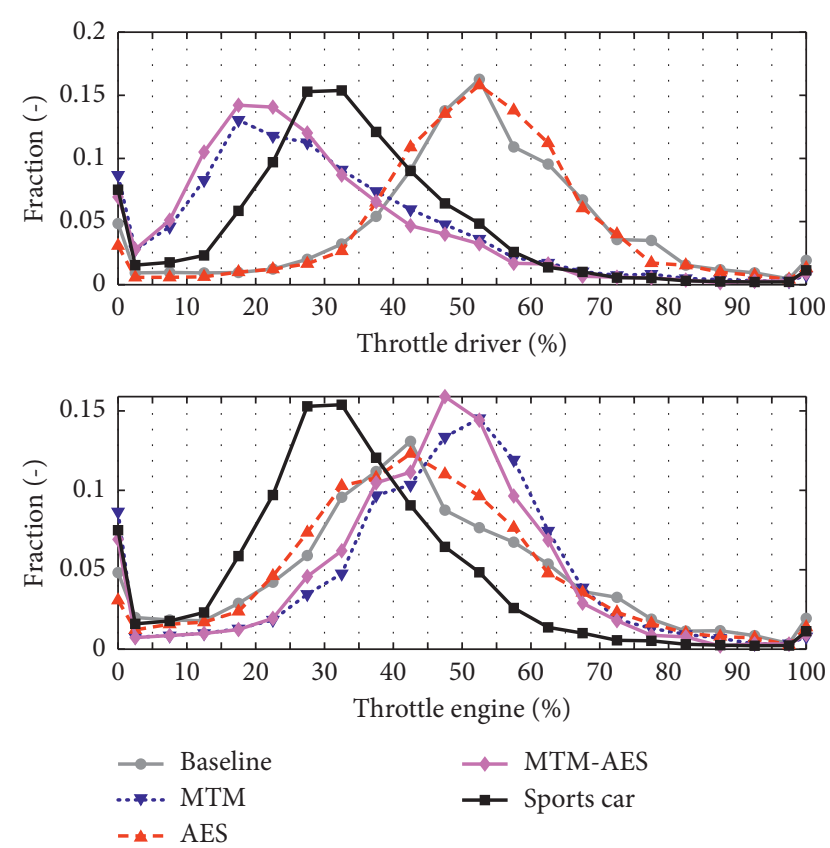

FIgURE 8: "Throttle driver" and "throttle engine" distribution of all participants for the curvy section. The fraction is plotted in the middle of each bin. The bin width is $5 \%$, where the first bin includes the scalar 0 , the second bin includes values greater than 0 and less than or equal to 5 , and so on. The last bin contains the scalar value of 100 . The sum of all fractions equals 1 for each condition.

decreased $S D$ speed and decreased mean absolute acceleration while driving through curves. In other words, AES induced more fluent driving behavior compared to Baseline.

3.5. Effects of Sports Car. Compared to the other four conditions, the Sports car yielded a high mean speed (on the straight section, not in the curvy section), a high $S D$ speed, and a high mean absolute acceleration. The highest mean acceleration during the $1 \mathrm{~s}$ acceleration phase was found for the Sports car. In other words, the Sports car resulted in sporty driving behavior.

\section{Discussion}

This study investigated the effects on perceived sportiness and driving behavior of Modified Throttle Mapping (MTM) and Artificial Engine Sound (AES) relative to a vehicle without these systems (Baseline) and a vehicle that offered increased engine power together with a sporty engine sound (Sports car). In the following, we discuss the results using the framework presented in Figure 1.

4.1. Artificial Engine Sound (AES). On a scale of 1 to 5, the mean perceived sportiness ratings for Baseline, MTM, AES, MTM-AES, and Sports car were 1.72, 1.84, 2.78, 2.97, and 3.91 , confirming the hypothesis that AES has a positive effect on perceived sportiness. AES was also clearly noticed and yielded high ratings of vehicle quickness and responsiveness. However, AES did not increase perceived sportiness to the

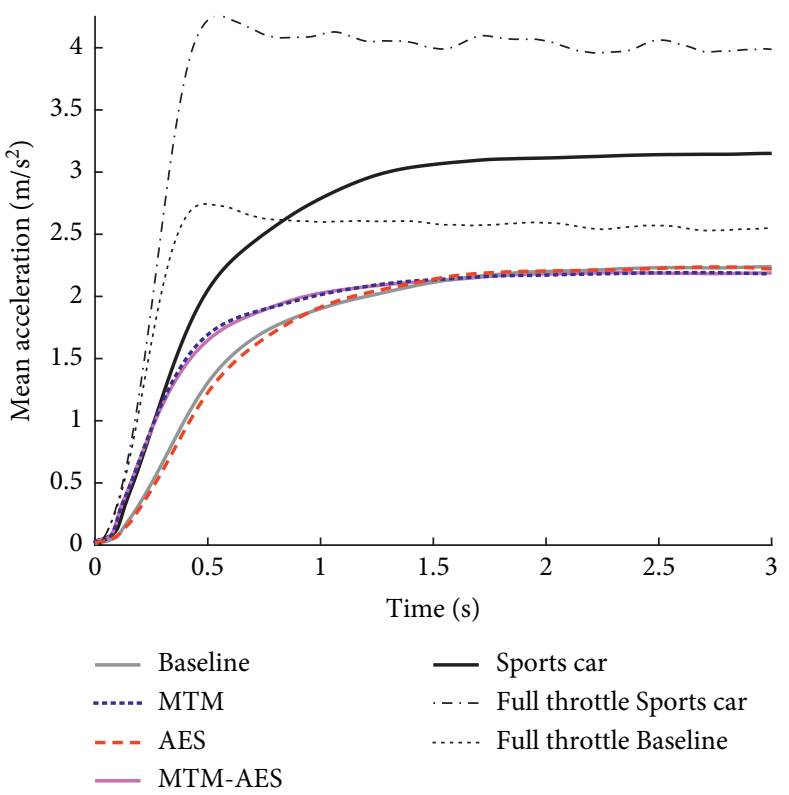

Figure 9: Mean vehicle acceleration for $0-60 \mathrm{~km} / \mathrm{h}$ acceleration phases, averaged over repetitions and participants during the first $3 \mathrm{~s}$. The "full throttle" lines are added as a reference.

extent of driving the Sports car. The principal component analysis complemented these findings, where a strong effect of AES and MTM-AES compared to Baseline was found on the PC1-Sportiness dimension, whereas PC2-Positive Affect scores were relatively unaffected by the experimental conditions. In other words, AES increased perceived sportiness without compromising comfort.

Regarding driving behavior, the AES and MTM-AES conditions yielded improved speed control (i.e., lower $S D$ speed) while driving through curves compared to Baseline. In other words, AES induced fluent rather than sporty driving behavior. These results can be interpreted using Figure 1. That is, a plausible explanation would be that, due to the more rapid change in virtual rpm for AES compared to Baseline, changes in engine torque were more readily noticed via the auditory feedback loop, which in turn improved speed control. These effects may be especially manifest in our fixedbase simulator, which did not provide vestibular feedback. Several previous studies agree on the importance of sound in vehicle speed control, but these studies typically investigated the impact of sound in driving tasks where the speedometer was not visible, possibly increasing the impact of auditory feedback [35]. Merat and Jamson [37] found an increase in speed variability compared to a Baseline condition, especially for a "no sound and no speedometer" condition. We found improvements in speed control even though the speedometer was permanently visible to the driver. With the presence of a speedometer, visual feedback delays can be expected to be smaller compared to when no speedometer is available, as the speedometer provides a direct reading. (and see Grether [69] where a digital reading yielded the fastest response times) Note that the speed variability benefits were statistically significant only for the curvy section, possibly because the visual demands from the roadway were higher during curve 
driving, as a result of which participants were unable to glance at the speedometer. In summary, the results indicate that AES increased perceived sportiness and improved speed control performance.

AES and MTM-AES did not significantly affect participants' mean driving speed relative to Baseline. This finding is inconsistent with the risk compensation mechanism outlined in the Introduction, based on which we hypothesized that the elevated virtual $\mathrm{rpm}$ would entice drivers to drive more slowly. Previous research has found that drivers slow down when provided with a more demanding environment (e.g., narrower lanes [69]; driving in fog [70]) or when driver assistance systems are disabled (e.g., [71, 72]). According to Elvik et al. [73], the degree of risk compensation can be predicted by the noticeability of the intervention and the "size of the engineering effect" of the intervention, where the latter is defined as the safety benefits caused by the intervention if the driver would not change his behavior. Although AES was clearly noticeable in our study according to the questionnaire outcomes, there was no engineering effect since AES did not affect the vehicle dynamics. In other words, AES did not offer a safety benefit, nor did it instigate drivers to drive faster.

Although no speed increase was found for MTM and AES, the Sports car did induce higher driving speeds and higher mean absolute acceleration than the other conditions. Put differently, the Sports car resulted in sporty driving behavior. This finding is not entirely self-evident. Previous cross-sectional research indicates that sporty vehicles are driven in a more risky manner than nonsporty vehicles, but the causal direction of this association remained unproven [74]. The present study showed that the Sports car yielded a $10 \mathrm{~km} / \mathrm{h}$ higher mean speed on the straight than MTM-AES, confirming that the availability of more engine power caused drivers to drive faster.

Earlier psychoacoustics research showed a decrease in comfort and acceptance for more sporty (rough; loud) sounds $[25,26,75]$, whereas we found no significant effects of comfort and acceptance for AES compared to Baseline. In fact, the Sports car yielded the highest self-reported desirability and likeability among the five conditions. The difference between our findings and previous research may have arisen because our participants were driving on a challenging trajectory that included curves. The sound, therefore, had a functional role (e.g., to aid in speed perception, see Figure 1), which may have contributed to improved comfort and acceptance (as also pointed out by [30]). Furthermore, our sample consisted of predominantly young males, who may be accepting of sporty sounds.

4.2. Modified Throttle Mapping (MTM). MTM caused substantially higher vehicle acceleration than Baseline, but this was not reflected in ratings of sportiness, quickness, acceleration effort, or engine responsiveness. These findings are inconsistent with our hypothesis that stated that MTM would increase perceived sportiness. The absence of effects of MTM in the self-report questionnaire suggests that participants may have hardly been aware of the altered throttle mapping. As pointed out above, in fixed-base simulators, acceleration can be sensed via the auditory and visual senses only [76-80]. It is recommended that future studies on the perception of MTM are conducted in a real vehicle, allowing for vestibular feedback. With reference to Figure 1, this would require an additional feedback loop in the form of motion feedback [69] for state-of-the-art manual control models incorporating vestibular motion feedback, see $[80])$.

Consistent with our hypothesis, participants adapted to the MTM by pressing the throttle less deeply in such a way that their mean driving speed was unaffected. In the context of Figure 1, drivers quickly adapted their "driver gain" to the increase in "MTM gain." Previous research concurs that human operators can adapt to different control gains [81, 82]. An early review by McRuer and Jex [44] showed that the range of optimum gains is wide, with only small deviations in self-reported handling qualities for $300-400 \%$ changes in the gain of the controlled vehicle. These findings are consistent with our study, which showed that drivers appeared to have hardly noticed the MTM.

Although MTM yielded the same mean "throttle engine" as the Baseline condition, it yielded a different "throttle engine" distribution. These findings can be explained directly by the throttle mapping, as depicted in Figure 4. For example, for the MTM condition, "throttle engine" values between 0 and $40 \%$ were obtained for only a small range of "throttle driver" (0-18\%).

Of note, MTM yielded a relatively high self-reported effort for speed control and throttle-release times that were about twice as high as the Baseline condition. It seems that the sensitive MTM pedal led to high pedal movements, as characterised by overshoot (i.e., high "throttle engine") and subsequent releasing of the pedal. A likely explanation is that high control gains amplify the influence of motor noise so that higher gain requires more corrective effort [81-83]. In other words, when driving with MTM, drivers fully adapted their "throttle engine" to achieve the same mean speed as Baseline, but at the cost of corrective pedal adjustments and effort. On the flip side, the advantage of a higher gain is that the required amplitude of limb movement is smaller.

4.3. MTM and AES Combined. A visual inspection of the results in Figure 6 and Table 3 reveals no clear $\mathrm{MTM} \times$ AES interaction effects. For example, the results for perceived sportiness (Q8) suggest that the effects of MTM and AES are additive, with "the whole" (i.e., effects of MTM-AES versus Baseline) being approximately equal to the "sum of its parts" (i.e., MTM versus Baseline and AES versus Baseline). The lack of interaction is supported by two-way repeated-measures ANOVAs using the results for Baseline, MTM, AES, and MTM-AES conditions as input. All 27 ANOVAs revealed a nonsignificant MTM $\times$ AES interaction effect $(p>0.05$ for each of the dependent variables, i.e., 14 self-reports and 13 performance measures). This lack of interaction may be surprising because the MTM-AES system is interactive in nature, as AES allows for a more direct perception of engine torque (see Figure 3). For example, MTM yielded 
higher throttle-released times than Baseline, something that should theoretically be better audible to participants when AES is enabled compared to when AES is disabled. Future research should employ larger sample sizes to be able to detect MTM $\times$ AES interactions that may exist.

4.4. Practical Implications and Recommendations. The current MTM implementation is easily translated to a real vehicle as it is based on a commercially available product that performs the same digital manipulation [84]. Similarly, electric vehicles could be equipped with an AES system similar to the one in this study. It should not be forgotten that a driving simulator itself provides only an illusion of driving [85]. In real electric vehicles, there are also ambient sounds (e.g., tires; wind) and vibrations, which may require active sound cancellation to provide a veridical synthetic sound. Furthermore, as recommended above, research in real vehicles is still needed.

It is noted that the current implementation of AES and MTM, although justified based on pilot testing, represents only one point in the large design space that exists. The current study involved pitch adjustments in engine sound, tested in acceleration, straight driving, and curve driving tasks. Other options would be to apply continuous auditory feedback to support braking, lane-keeping, car-following, or automation mode awareness (e.g., [86, 87]).

Apart from investigating other sounds and throttle mappings, it would be interesting to investigate how other technologies affect perceived sportiness and driving behavior. Sport modes commonly use red ambient light $[7,88,89]$ and a sporty instrumented cluster (e.g., see [90-92]). Other techniques to increase perceived sportiness are to increase steering torques and center point emphasis of the steering system [93], shortening the gear shift stroke [40], changing the seat so that it has high side supports [94], and recreating the road feedback between front-wheel slip and steering force that has been lost due to power steering [95].

\section{Conclusions}

This study was concerned with examining how drivers perceive and respond to electric-vehicle parameter adjustments that intend to provide a sporty driving experience. Modified Throttle Mapping (MTM) and Artificial Engine Sound (AES) were tested, systems that affect the "gain" of the accelerator pedal and the auditory feedback provided to drivers, respectively, and do not enhance the car's performance envelope. The results showed the following effects relative to the Baseline condition:

(i) AES increased perceived sportiness, whereas MTM did not

(ii) AES and MTM did not affect perceived comfort

(iii) AES yielded improved speed control in curves

(iv) MTM increased vehicle acceleration from a standstill (v) AES and MTM yielded average driving speeds that were comparable to Baseline

(vi) No AES $\times$ MTM interaction effects were found

These novel findings may have utility for vehicle manufacturers. Throttle mapping (i.e., MTM gain as depicted in Figure 1) is a component of every vehicle, but so far, no research seems to have examined its effects on driving behavior and perception. Research into the effects of sound on driving behavior has so far mainly focused on the presence/absence or volume of sound and not on sound enhancement as could be applied in electric vehicles. Furthermore, research on vehicle sound has to date been predominantly conducted in listening rooms, a setting which, as argued in the Introduction, is not realistic. In listening rooms, sporty sounds tend to be perceived as uncomfortable, whereas in our study, sporty sounds were not seen as uncomfortable but rather contributed to improved speed control performance.

In addition to its applied value, this study offers fundamental insights into human perception and behavior. The current findings were interpreted using principles from manual control theory, as shown in Figure 1, where we highlighted the role of driver adaptation (drivers fully adapted their own gain to the increased MTM gain) and feedback loops (drivers used the artificial sound as a feedback channel that aided in speed control). Furthermore, no risk compensation occurred; that is, in the context of Figure 1, the "desired speed" remained unaffected.

In more general terms, our study indicates that findings from complex tasks, such as driving, can be interpreted with the help of qualitative representations from control theory, a notion emphasized by several psychologists and human factors scientists before [96-98] which is still undervalued [99].

\section{Data Availability}

The data, analysis scripts, and videos of the experiment can be downloaded via the following link: https://doi.org/10. $4121 / 16644697$.

\section{Conflicts of Interest}

The authors declare that they have no conflicts of interest.

\section{Acknowledgments}

The authors would like to thank Joris Giltay for his help in implementing the experiment in the new driving simulator.

\section{References}

[1] T. Rothengatter, "Risk and the absence of pleasure: a motivational approach to modelling road user behaviour," Ergonomics, vol. 31, no. 4, pp. 599-607, 1988.

[2] JATO, "European new car market starts 2021 with record market share for SUVs," 2021, https://www.jato.com/europeannew-car-market-starts-2021-with-record-market-share-for-suv. 
[3] Audi, "Driving dynamic. Audi drive select," 2021, https:// ownersmanuals2.com/audi/q7-2020-owners-manual-75410 /page-110.

[4] BMW, "Drive modes in detail," 2021, https://ownersmanuals2. com/bmw-auto/x5-2021-owners-manual-80671/page-161.

[5] Mercedes-Benz, "Driving modes," 2021, https://moba.i. daimler.com/markets/ece-row/baix/cars/177.0_mbux-high_20 20_a/en_GB/page/ID_9ec704d53993f553354ae365263f0b2f-1d 3ef3273993f563354ae36502733902-en-GB.html.

[6] Porsche, "Model overview. Panamera models," 2021, https:// www.porsche.com/usa/models/panamera/panamera-e-hybr id-models/drivechassis/sport-mode.

[7] Renault, "Multi-sense," 2021, https:/gb.e-guide.renault.com/ eng/easy-link/MULTI-SENSE.

[8] Volvo, "V90 cross country," Drive modes, https://www.volvocars. com/en-th/support/manuals/v90-cross-country/2019w17/startin g-and-driving/drive-modes/drive-modes, 2021.

[9] L. De Novellis, A. Sorniotti, and P. Gruber, "Driving modes for designing the cornering response of fully electric vehicles with multiple motors," Mechanical Systems and Signal Processing, vol. 64-65, pp. 1-15, 2015.

[10] T. Melman, J. De Winter, X. Mouton, A. Tapus, and D. Abbink, "How do driving modes affect the vehicle's dynamic behaviour? Comparing Renault's Multi-Sense sport and comfort modes during on-road naturalistic driving," Vehicle System Dynamics, vol. 59, no. 4, pp. 485-503, 2021.

[11] Y. Shibahata, "Progress and future direction of chassis control technology," Annual Reviews in Control, vol. 29, no. 1, pp. 151-158, 2005.

[12] T. Shinagawa, M. Kudo, W. Matsubara, and T. Kawai, "The new Toyota 1.2-liter ESTEC turbocharged direct injection gasoline engine (Technical Paper No. 2015-01-1268)," SAE International, 2015.

[13] K. Wehbi, D. Bestle, and J. Beilharz, "Automatic calibration process for optimal control of clutch engagement during launch," Mechanics Based Design of Structures and Machines, vol. 45, no. 4, pp. 507-522, 2017.

[14] Y. Furukawa, N. Yuhara, S. Sano, H. Takeda, and Y. Matsushita, "A review of four-wheel steering studies from the viewpoint of vehicle dynamics and control," Vehicle System Dynamics, vol. 18, no. 1-3, pp. 151-186, 1989.

[15] P. Herold and M. Wallbrecher, "All-wheel steering," in Steering Handbook, M. Harrer and P. Pfeffer, Eds., Springer, Cham, Switzerland, pp. 493-512, 2017.

[16] M. Ambrosino, L. Lubrano, F. Sciacca, P. Giorgi, and L. Ferla, "DualMode sporty exhaust development (Technical Paper No. 2011-01-0926)," SAE International, 2011.

[17] A. P. Jackson, "A comparison between active and passive approaches to the sound quality tuning of a high performance vehicle (Technical Paper No. 2013-01-1878)," SAE International, 2013.

[18] T. Bräunl, "Synthetic engine noise generation for improving electric vehicle safety," International Journal of Vehicle Safety, vol. 6, no. 1, pp. 1-8, 2012.

[19] Y. Fang and T. Zhang, "Sound quality investigation and improvement of an electric powertrain for electric vehicles," IEEE Transactions on Industrial Electronics, vol. 65, pp. 1149-1157, 2017.

[20] K. Govindswamy and G. Eisele, "Sound character of electric vehicles (No. 2011-01-1728)," SAE Technical Paper, 2011.

[21] D. Min, B. Park, and J. Park, "Artificial engine sound synthesis method for modification of the acoustic characteristics of electric vehicles," Shock and Vibration, 2018.
[22] P. Nyeste and M. S. Wogalter, "On adding sound to quiet vehicles," Proceedings of the Human Factors and Ergonomics Society Annual Meeting, vol. 52, no. 21, pp. 1747-1750, 2008.

[23] S. M. Faas and M. Baumann, "Pedestrian assessment: is displaying automated driving mode in self-driving vehicles as relevant as emitting an engine sound in electric vehicles?" Applied Ergonomics, vol. 94103425 pages, 2021.

[24] E. Karaaslan, M. Noori, J. Lee, L. Wang, O. Tatari, and M. Abdel-Aty, "Modeling the effect of electric vehicle adoption on pedestrian traffic safety: an agent-based approach," Transportation Research Part C: Emerging Technologies, vol. 93, pp. 198-210, 2018.

[25] J. Krüger, F. Castor, and A. Müller, "Psychoacoustic investigation on sport sound of automotive tailpipe noise," Fortschritte der Akustik-DAGA, pp. 233-234, Eberspächer Group, Strasbourg, France, 2004.

[26] G. Kwon, H. Jo, and Y. J. Kang, "Model of psychoacoustic sportiness for vehicle interior sound: excluding loudness," Applied Acoustics, vol. 136, pp. 16-25, 2018.

[27] P. A. Jennings, G. Dunne, R. Williams, and S. Giudice, “Tools and techniques for understanding the fundamentals of automotive sound quality," Proceedings of the Institution of Mechanical Engineers, Part D: Journal of Automobile Engineering, vol. 224, no. 10, pp. 1263-1278, 2010.

[28] D. Park, S. Park, W. Kim, I. Rhiu, and M. H. Yun, "A comparative study on subjective feeling of engine acceleration sound by automobile types," International Journal of Industrial Ergonomics, vol. 74, Article ID 102843, 2019.

[29] R. Bisping, "Emotional effect of car interior sounds: pleasantness and power and their relation to acoustic key features," SAE Transactions, vol. 104, pp. 2207-2213, 1995.

[30] E. Hellier, A. Naweed, G. Walker, P. Husband, and J. Edworthy, "The influence of auditory feedback on speed choice, violations and comfort in a driving simulation game," Transportation Research Part F: Traffic Psychology and Behaviour, vol. 14, no. 6, pp. 591-599, 2011.

[31] A. Zeitler and P. Zeller, "Psychoacoustic modelling of sound attributes (No. 2006-01-0098)," SAE Technical Paper, 2006.

[32] M. S. Horswill and F. P. McKenna, "The development, validation, and application of a video-based technique for measuring an everyday risk-taking behavior: drivers' speed choice," Journal of Applied Psychology, vol. 84, no. 6, pp. 977-985, 1999.

[33] L. Evans, "Speed estimation from a moving automobile," Ergonomics, vol. 13, no. 2, pp. 219-230, 1970.

[34] M. S. Horswill and A. M. Plooy, "Auditory feedback influences perceived driving speeds," Perception, vol. 37, no. 7, pp. 1037-1043, 2008.

[35] S. Denjean, V. Roussarie, R. Kronland-Martinet, S. Ystad, and J. L. Velay, "How does interior car noise alter driver's perception of motion? Multisensory integration in speed perception," in Proceedings of the Acoustics 2012 Nantes Conference, S. F. d'Acoustique, Ed., Nantes, France, April 2012.

[36] R. C. McLane and W. W. Wierwille, "The influence of motion and audio cues on driver performance in an automobile simulator," Human Factors: The Journal of the Human Factors and Ergonomics Society, vol. 17, no. 5, pp. 488-501, 1975.

[37] N. Merat and H. Jamson, "A driving simulator study to examine the role of vehicle acoustics on drivers' speed perception," in Proceedings of the Sixth International Driving Symposium on Human Factors in Driver Assessment, Training and Vehicle Design, pp. 226-232, Lake Tahoe, CA, USA, June 2011. 
[38] T. D. Barker, "Engine mapping techniques," International Journal of Vehicle Design, vol. 3, pp. 142-152, 1982.

[39] T. Holliday, A. J. Lawrance, and T. P. Davis, "Engine-mapping experiments: a two-stage regression approach," Technometrics, vol. 40, no. 2, pp. 120-126, 1998.

[40] M. Hosoda, "Power train for a new compact sporty hybrid vehicle (Technical Paper No. 2010-01-1095)," SAE International, 2010.

[41] P. Schoeggl, E. Ramschak, and E. Bogner, "On-board optimization of driveability character depending on driver style by using a new closed loop approach (No. 2001-01-0556)," SAE Technical Paper, 2001.

[42] R. Boris, C. Vermillion, and K. Butts, "A comparative analysis of electronic pedal algorithms using a driver-in-the-loop simulator and system identification of driver behavior," in Proceedings of the 2010 American Control Conference, pp. 682-687, Baltimore, MD, USA, 2010.

[43] M. Mulder, J. J. A. Pauwelussen, M. M. Van Paassen, M. Mulder, and D. A. Abbink, "Active deceleration support in car following," IEEE Transactions on Systems, Man, and Cybernetics - Part A: Systems and Humans, vol. 40, no. 6, pp. 1271-1284, 2010.

[44] D. T. McRuer and H. R. Jex, "A review of quasi-linear pilot models," IEEE Transactions on Human Factors in Electronics, vol. HFE-8, no. 3, pp. 231-249, 1967.

[45] D. H. Weir and K. C. Chao, "Review of control theory models for directional and speed control," in Modelling Driver Behaviour in Automotive Environments: Critical Issues in Driver Interactions with Intelligent Transport Systems, P. Cacciabue, Ed., Springer, New York, NY, USA, pp. 293-311, 2007.

[46] D. T. McRuer, R. W. Allen, D. H. Weir, and R. H. Klein, "New results in driver steering control models," Human Factors: The Journal of the Human Factors and Ergonomics Society, vol. 19, no. 4, pp. 381-397, 1977.

[47] M. Sivak, "The information that drivers use: is it indeed $90 \%$ visual?" Perception, vol. 25, pp. 1081-1089, 1996.

[48] R. J. Jagacinski and J. M. Flach, Control Theory for Humans-Quantitative Approaches to Modeling Performance, Lawrence Erlbaum, Mahwah, NJ, USA, 2003.

[49] R. Hosman and H. Stassen, "Pilot's perception and control of aircraft motions," IFAC Proceedings Volumes, vol. 31, no. 26, pp. 311-316, 1998.

[50] J. F. Larish and J. M. Flach, "Sources of optical information useful for perception of speed of rectilinear self-motion," Journal of Experimental Psychology: Human Perception and Performance, vol. 16, no. 2, pp. 295-302, 1990.

[51] R. Fuller, "Towards a general theory of driver behaviour," Accident Analysis \& Prevention, vol. 37, no. 3, pp. 461-472, 2005.

[52] T. Melman, D. A. Abbink, M. M. Van Paassen, E. R. Boer, and J. C. F. De Winter, "What determines drivers' speed? A replication of three behavioural adaptation experiments in a single driving simulator study," Ergonomics, vol. 61, no. 7, pp. 966-987, 2018.

[53] G. J. S. Wilde, "Risk homeostasis theory: an overview," Injury Prevention, vol. 4, no. 2, pp. 89-91, 1998.

[54] N. Beckers, O. Siebinga, J. Giltay, and A. Van der Kraan, "JOAN, a human-automated vehicle experiment framework (Version 1.0) [Computer software]," 2021, https://github. com/tud-hri/joan.

[55] A. Dosovitskiy, G. Ros, F. Codevilla, A. Lopez, and V. Koltun, "CARLA: an open urban driving simulator," in Proceedings of the 1st Annual Conference on Robot Learning, pp. 1-16,
November 2017, http://proceedings.mlr.press/v78/ dosovitskiy17a/dosovitskiy17a.pdf.

[56] Unreal, "Unreal engine 4 documentation," 2021, https://docs. unrealengine.com/en-US/index.html.

[57] Soundsnap, "Volkswagen Golf V 1.6 FSI engine regular driving with slow acceleration mono," 2021a, https://www. soundsnap.com/volkswagen_golf_v_1_6_fsi_engine_regular _driving_with_slow_acceleration_mono_wav.

[58] Soundsnap, "Audi A4 B8 20TDI engine 3k RPM loop mono," 2021b, https://www.soundsnap.com/audi_a4_b8_20tdi_engine_ 3k_rpm_loop_mono_wav.

[59] J. D. Van der Laan, A. Heino, and D. De Waard, “A simple procedure for the assessment of acceptance of advanced transport telematics," Transportation Research Part C: Emerging Technologies, vol. 5, no. 1, pp. 1-10, 1997.

[60] L. Aarts and I. Van Schagen, "Driving speed and the risk of road crashes: a review," Accident Analysis \& Prevention, vol. 38, no. 2, pp. 215-224, 2006.

[61] X. Pei, S. C. Wong, and N. N. Sze, "The roles of exposure and speed in road safety analysis," Accident Analysis \& Prevention, vol. 48, pp. 464-471, 2012.

[62] E. Ericsson, "Independent driving pattern factors and their influence on fuel-use and exhaust emission factors," Transportation Research Part D: Transport and Environment, vol. 6, no. 5, pp. 325-345, 2001.

[63] C. Marina Martinez, M. Heucke, F.-Y. Wang, B. Gao, and D. Cao, "Driving style recognition for intelligent vehicle control and advanced driver assistance: a survey," IEEE Transactions on Intelligent Transportation Systems, vol. 19, no. 3, pp. 666-676, 2018.

[64] M. Houtenbos, J. C. F. De Winter, A. R. Hale, P. A. Wieringa, and M. P. Hagenzieker, "Concurrent audio-visual feedback for supporting drivers at intersections: a study using two linked driving simulators," Applied Ergonomics, vol. 60, pp. 30-42, 2017.

[65] H. Yeo, S. E. Shladover, H. Krishnan, and A. Skabardonis, "Microscopic traffic simulation of vehicle-to-vehicle hazard alerts on freeway," Transportation Research Record: Journal of the Transportation Research Board, vol. 2189, no. 1, pp. 68-77, 2010.

[66] R. D. Morey, "Confidence intervals from normalized data: a correction to Cousineau (2005)," Tutorials in Quantitative Methods for Psychology, vol. 4, no. 2, pp. 61-64, 2008.

[67] G. Cumming and S. Finch, "Inference by eye: confidence intervals and how to read pictures of data," American Psychologist, vol. 60, no. 2, pp. 170-180, 2005.

[68] W. F. Grether, "Instrument reading. I. The design of longscale indicators for speed and accuracy of quantitative readings," Journal of Applied Psychology, vol. 33, no. 4, pp. 363-372, 1949.

[69] T. Melman, S. Kolekar, E. Hogerwerf, and D. Abbink, "How road narrowing impacts the trade-off between two adaptation strategies: reducing speed and increasing neuromuscular stiffness," in Proceedings of the 2020 IEEE International Conference on Systems, Man, and Cybernetics, pp. 3235-3240, Toronto, Canada, October 2020.

[70] J. O. Brooks, M. C. Crisler, N. Klein et al., "Speed choice and driving performance in simulated foggy conditions," Accident Analysis \& Prevention, vol. 43, no. 3, pp. 698-705, 2011.

[71] W. Janssen and L. Nilsson, An Experimental Evaluation of InVehicle Collision Avoidance Systems (GIDS Deliverable MAN2), Traffic Research Centre, University of Groningen, Haren, Netherlands, 1993. 
[72] T. Melman, J. C. F. De Winter, and D. A. Abbink, "Does haptic steering guidance instigate speeding? A driving simulator study into causes and remedies," Accident Analysis \& Prevention, vol. 98, pp. 372-387, 2017.

[73] R. Elvik, A. Høye, T. Vaa, and M. Sørensen, "Basic concepts of road safety research," The Handbook of Road Safety Measures, Emerald Group Publishing Limited, Bingley, UK, pp. 81-98, 2009.

[74] M. S. Horswill and M. E. Coster, "The effect of vehicle characteristics on drivers' risk-taking behaviour," Ergonomics, vol. 45, no. 2, pp. 85-104, 2002.

[75] Y. S. Wang, G. Q. Shen, and Y. F. Xing, "A sound quality model for objective synthesis evaluation of vehicle interior noise based on artificial neural network," Mechanical Systems and Signal Processing, vol. 45, no. 1, pp. 255-266, 2014.

[76] E. R. Boer, A. R. Girshik, T. Yamamura, and N. Kuge, "Experiencing the same road twice: a driver centered comparison between simulation and reality," in Proceedings of the Driving Simulation Conference Europe, Paris, France, 2000.

[77] S. De Groot, M. Mulder, P. A. Wieringa, and P. A. Wieringa, "Nonvestibular motion cueing in a fixed-base driving simulator: effects on driver braking and cornering performance," Presence: Teleoperators and Virtual Environments, vol. 20, no. 2, pp. 117-142, 2011.

[78] J. Greenberg, B. Artz, and L. Cathey, "The effect of lateral motion cues during simulated driving," in Proceedings of DSC North America, Dearborn, MI, USA, October 2003.

[79] D. McRuer, D. H. Weir, and L. Cathey, "Theory of manual vehicular control," Ergonomics, vol. 12, pp. 599-633, 1969.

[80] M. Mulder, D. M. Pool, D. A. Abbink et al., "Manual control cybernetics: state-of-the-art and current trends," IEEE Transactions on Human-Machine Systems, vol. 48, pp. 468485, 2017.

[81] J. C. F. De Winter and S. De Groot, "The effects of controldisplay gain on performance of race car drivers in an isometric braking task," Journal of Sports Sciences, vol. 30, no. 16, pp. 1747-1756, 2012.

[82] W. L. Jenkins and M. B. Connor, "Some design factors in making settings on a linear scale," Journal of Applied Psychology, vol. 33, no. 4, pp. 395-409, 1949.

[83] A. Chapanis and R. G. Kinkade, "Design of controls," in Human Engineering Guide to Equipment Design, H. P. van Cott and R. G. Kinkade, Eds., pp. 345-379, American Institute for Research, Washington, DC, 1972.

[84] DTE Systems, "The PedalBox: The throttle response," 2021, https://www.pedalbox.com/en/product/response.

[85] P. Hancock, "The future of simulation," in Human Factors in Simulation and Training, D. Vincenzi, J. Wise, M. Mouloua, and P. A. Hancock, Eds., CRC Press, Taylor \& Francis, Boca Raton, FL, USA, pp. 169-186, 2009.

[86] P. Bazilinskyy, P. Larsson, E. Johansson, and J. C. F. De Winter, "Continuous auditory feedback on the status of adaptive cruise control, lane deviation, and time headway: an acceptable support for truck drivers?" Acoustical Science and Technology, vol. 40, no. 6, pp. 382-390, 2019.

[87] L. Bringoux, J. Monnoyer, P. Besson et al., "Influence of speed-related auditory feedback on braking in a 3D-driving simulator," Transportation Research Part F: Traffic Psychology and Behaviour, vol. 44, pp. 76-89, 2017.

[88] M. G. Helander, H. M. Khalid, T. Y. Lim, H. Peng, and $\mathrm{X}$. Yang, "Emotional needs of car buyers and emotional intent of car designers," Theoretical Issues in Ergonomics Science, vol. 14, no. 5, pp. 455-474, 2013.
[89] M. Nieke, J. Mauro, C. Seidl, and I. C. Yu, "User profiles for context-aware reconfiguration in software product lines," in Leveraging Applications of Formal Methods, Verification and Validation: Discussion, Dissemination, Applications. ISoLA 2016. Lecture Notes in Computer Science, T. Margaria and B. Steffen, Eds., vol. 9953, pp. 563-578, Springer, Cham, Switzerland, 2016.

[90] T. Jindo and K. Hirasago, "Application studies to car interior of Kansei engineering," International Journal of Industrial Ergonomics, vol. 19, no. 2, pp. 105-114, 1997.

[91] G. Jung, S. M. Kim, S. Y. Kim, E. S. Jung, and S. Park, "Effects of design factors of the instrument cluster panel on consumers' affection," in Proceedings of the International MultiConference of Engineers and Computer Scientists, vol. 3, Hong Kong, March 2010.

[92] J. F. Petiot, C. C. Francisco, and B. Ludivine, "A comparison of conjoint analysis and interactive genetic algorithms for the study of product semantics," in Proceedings of the 20th International Conference on Engineering Design (ICED 15) Vol 5: Design Methods and Tools-Part 1, Milan, Italy, July 2015.

[93] S. Fankem and S. Müller, "A new model to compute the desired steering torque for steer-by-wire vehicles and driving simulators," Vehicle System Dynamics, vol. 52, no. S1, pp. 251-271, 2014.

[94] I. Kamp, "The influence of car-seat design on its character experience," Applied Ergonomics, vol. 43, no. 2, pp. 329-335, 2012.

[95] A. Uselmann, K. M. Krüger, C. Bittner, and G. Rivera, "Innovative software functions to operate electric power steering systems in sports cars-unterstützungskraftregelung (UKR)," in Proceedings of the 6th International Munich Chassis Symposium 2015, P. Pfeffer, Ed., pp. 423-441, Springer Vieweg, Wiesbaden, Germany, June 2015.

[96] J. M. Flach, "Control with an eye for perception: precursors to an active psychophysics," Ecological Psychology, vol. 2, no. 2, pp. 83-111, 1990.

[97] R. J. Jagacinski, “A qualitative look at feedback control theory as a style of describing behavior," Human Factors: The Journal of the Human Factors and Ergonomics Society, vol. 19, no. 4, pp. 331-347, 1977.

[98] T. B. Sheridan, "Driver distraction from a control theory perspective," Human Factors: The Journal of the Human Factors and Ergonomics Society, vol. 46, no. 4, pp. 587-599, 2004.

[99] W. Mansell and R. S. Marken, "The origins and future of control theory in psychology," Review of General Psychology, vol. 19, no. 4, pp. 425-430, 2015. 